\title{
Performance study of ground-based infrared Bracewell interferometers
}

\section{Application to the detection of exozodiacal dust disks with GENIE (Corrigendum)}

\author{
O. Absil ${ }^{1, \star}$, R. den $\operatorname{Hartog}^{2}$, P. Gondoin ${ }^{2}$, P. Fabry ${ }^{2}$, R. Wilhelm ${ }^{3}$, P. Gitton ${ }^{3}$, and F. Puech ${ }^{3}$ \\ 1 Institut d'Astrophysique et de Géophysique, Université de Liège, 17 Allée du Six Août, 4000 Sart-Tilman, Belgium \\ e-mail: absil@astro.ulg.ac.be \\ 2 Science Payloads and Advanced Concepts Office, ESA/ESTEC, postbus 299, 2200 AG Noordwijk, The Netherlands \\ 3 European Southern Observatory, Karl-Schwarzschild-Str. 2, 85748 Garching bei München, Germany
}

A\&A 448, 787-800 (2006), DOI: 10.1051/0004-6361:20053516

Key words. instrumentation: high angular resolution - instrumentation: interferometers - techniques: interferometric circumstellar matter - planetary systems - errata, addenda

In p. 789 of the published article, Eq. (3) should be corrected to

$$
N_{\mathrm{LD}}=1 / \rho_{\mathrm{LD}}=\frac{\pi^{2}}{4}\left(\frac{B \theta_{\mathrm{LD}}}{\lambda}\right)^{2}\left(1-\frac{7 u_{\lambda}}{15}\right)\left(1-\frac{u_{\lambda}}{3}\right)^{-1},
$$

with $\theta_{\mathrm{LD}}$ the limb-darkened stellar angular radius. When equating this corrected equation to Eq. (2) of Absil et al. (2006), one can derive the expression of the ratio $\theta_{\mathrm{LD}} / \theta_{\mathrm{UD}}$ between the limb-darkened and uniform-disk angular radii that produce the same null depth, which now agrees with Eq. (5) of Hanbury Brown et al. (1974).

Acknowledgements. The authors are grateful to Bertrand Mennesson for suggesting this correction.

\section{References}

Absil, O., den Hartog, R., Gondoin, P., et al. 2006, A\&A, 448, 787

Hanbury Brown, R., Davis, J., Lake, R. J. W., \& Thompson, R. J. 1974, MNRAS, 167, 475

* FNRS Postdoctoral Researcher. 